Distribution Category: Atomic, Molecular, and Chemical Physics (UC-411)

ARGONNE NATIONAL LABORATORY

9700 South Cass Avenue

Argonne, Illinois 60439

ANL/APS;'T'B--8

DE93 008835

ANL/APS/TB-8

\title{
CAT Questions and Answers
}

Experimental Facilities Division

Advanced Photon Source

February 1993

work sponsored by

U.S. DEPARTMENT OF ENERGY

Office of Energy Research 


\section{Introduction}

This document, prepared in February 1993, addresses the most common questions asked by APS Collaborative Access Teams (CATs). The answers represent the best judgment on the part of the APS at this time. In some cases, details are provided in separate documents to be supplied by the APS. Some of the answers are brief because details are not yet available.

The questions are separated into five categories representing different aspects of CAT interactions with the APS: (1) Memorandum of Understanding (MOU), (2) CAT Beamline Review and Construction, (3) CAT Beamline Safety, (4) CAT Beamline Operations, and (5) Miscellaneous.

The APS plans to generate similar documents as needed to both address new questions and clarify answers to present questions. 


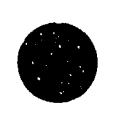

$\bullet$

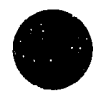




\section{Memorandum of Understanding (MOU)}

\section{Q. When will a draft version of the MOU will be available?}

A. A draft was distributed to all CAT Directors during the second week of January 1993. This draft was produced in response to input from the advisory subcommittee appointed by the APSUO Steering Committee. An additional copy appears in Appendix 1 for reference.

Q. What are the requirements that lead to the MOU?

A. As detailed in a number of previous documents, the following steps need to be completed before a CAT is ready to sign an MOU.

1. The CAT must have an approved scientific proposal.

2. The CAT must have an approved Beamine Conceptual Design Report.

3. The CAT must have an APS-approved Management Plan.

4. The CAT must have some documented evidence of financial backing from the sponsor(s) to meet the cost of beamline(s) construction.

Q. When will the CATs learn about the status of their Conceptual Design reviews?

A. The results of the reviews were mailed to all reviewed CATs in January 1993.

Q. Can the CAT change the Conceptual Design after it has been approved?

A. Minor changes in the concept will occur in the course of developing an engineering design of the beamline. However, major changes in the conceptual design should be reported to the APS to assure that such changes are acceptable. If major changes occur in the concept, the APS might seek advice from the Proposal Evaluation Board or its appointed review panel.

Q. What are the current requirements of the CAT Management Plan?

A. The basic elements of the Management Plan have not changed from those given to CATs in November 1990 (in Guidelines and Forms for Proposals to Establish Collaborative Access Teams at the Advanced Photon Source). 
A copy of these guidelines appears in Appendix 2. More detailed guidance on some of the elements will be presented at the 1993 CAT Workshop.

\section{Q. Who will approve the CAT Management Plan and when?}

A. After the Management Plan is submitted, the APS will work with the CAT to ensure that all the aspects of the plan are workable. A Management Plan Review Group has been organized for this purpose.

Q. Can the Management Plan be changed by the CAT after it is approved by the APS?

A. It is normal for a CAT to alter or improve the Management Plan as the activities of the CAT members become better defined. This document should be considered a "living" document. Changes should be reported to the APS on a timely basis.

Q. Are there any circumstances under which changes in an MOU might be required?

A. 1. A change in a CAT's institutional membership.

2. A change in the number of sectors allotted to a CAT. 


\section{CAT Beamline Review and Construction}

\section{Q. Describe the CAT beamline review process.}

A. 1. Obtain APS approval of the Conceptual Design.

2. Prepare a Preliminary Design. General guidance for the preparation of a Preliminary Design is provided in Appendix 3. It should be understood that development of a Preliminary Design will depend on the technical and scientific needs of the CAT. Hence, while following the general guidance, we suggest that the CAT consult on a regular basis with the APS in order to optimally use the resources from the APS Design Exchange and those available on the APS Experiment Floor. The interaction process will also assure that adequate attention is paid to the safety aspects of the design from its inception. As a part of the Preliminary Design, the CAT must develop a realistic cost and schedule estimate to build the proposed beamlines; again, the APS can provide guidance to each CAT on a one-on-one basis.

3. Obtain APS approval of the Preliminary Design.

4. Prepare the Final Design. This is a complete engineering design of the beamlines; usually very few changes are made in the design after this stage. The technical drawings are of suitable quality to begin construction. In this design, all aspects of safety are fully included in the design drawings. At this level of design, a cost estimate should again be performed.

5. Obtain APS approval of the Final Design.

Q. When can a CAT begin procurement, fabrication, and assembly of beamline components?

A. Anytime after the APS has approved the final beamline design from the standpoint of safety.

Q. Is there a timeline set by the APS for the above design process?

A. The APS will not set timelines for the CATs. However, to assure a timely start for construction, it is essential that each CAT develop and adhere to a timeline. For general guidance, an acceptable timeline appears as Appendix 4. 
Q. Is the APS going to mandate the use of standard and modular components in the beamline design?

A. The APS has developed designs for standard and modular components of a beamline for use by CATs. Some of these designs have already been subjected to performance analysis, safety analysis, design reviews, and safety reviews. However, a CAT might not choose to use the APS standard component designs. In that case, the APS will be required to subject CAT component designs to required performance and safety reviews. It is important for each CAT to realize that the use of APS standard and modular component designs will greatly reduce design costs to the CAT. If the standard and modular component designs are not optimally suited for a particular CAT's beamline, it is suggested that a CAT choose to modify the standard component design to ineet the specific requirements rather than develop a brand-new design.

Q. Will the APS beamline standard components have safety approval?

A. Yes, the final design of the standard components will be reviewed and approved. However, the safety approval of individual components is only one part of the total beamline safety. The safety review of the integrated beamline will be addressed as a part of the Final Design review.

Q. Will the APS provide help to the CAT during the Preliminary and Final Design activities? How will this be arranged?

A. Whatever help and guidance that the APS can provide is available to all CATs. The APS User Technical Interface Group will arrange the necessary interactions.

Q. How will the CATs generate the cost estimates of the beamlines requested as a part of the Preliminary Design?

A. First, it is important to realize that this exercise will help the CAT to obtain the required support from the funding source because it will help validate its request. In arriving at a cost estimate, the CAT can use its own experience with such activities. Also, the APS will work with the CATs to assess costs of major technical items or the standard and modular components. 


\section{Q. The CATs need much more information related to the availability of utilities and their location in the Experiment Hall. How will the CATs access such APS design information?}

A. The APS designs of the utilities in the Experiment Hall are now at the installation stage. These will be outlined in a separate document to be distributed to all CATs and will also be described at the Cat Workshop in February 1993. The documentation will cover electric power, deionized water, chilled water, laboratory water, exhausts for toxic and nontoxic gases, networks, Experiment Hall temperature control, additional airconditioning capabilities, cable trays, etc.

Q. When will the CATs receive firmer guidance from the APS on radiation shielding and ozone control?

A. Documents on these topics are now being prepared for distribution; these topics will also be presented at the CAT Workshop in February 1993.

Q. What is the redundancy factor in the safety of the beam stops for the white and the monochromatic radiation?

A. This is described in a separate safety document that will be distributed to all the CATs. The subject will also be addressed at the CAT Workshop in February 1993.

Q. When will the CATs learn about beamline interlock philosophy and lingic?

A. A preliminary interlock philosophy and logic description for the beamline and its front end as related to personnel and equipment safety will be presented to the CATs during the CAT workshop in February 1993.

Q. Are there fire codes that are applicable to CAT Experimental Stations (hutches)?

A. No, the required fire safety codes have been met by the APS in the Experiment Hall. Special considerations may be required to address the needs of specific experiments, but this is not part of the beamline safety design.

Q. Will APS provide survey monuments on the APS Experiment Floor?

A. Yes. These monuments will be provided prior to CAT beamline installation work. 
Q. Can the CATs plan on drilling holes in the APS Experiment Floor for erecting supports?

A. Yes, but the dimensions and the depth of the holes must be reviewed by the APS. This will take place as a part of the Final Design review of the beamlines.

Q. During beamline installation by the CAT, will the APS provide major items such as fork-lifts, cranes, etc.?

A. The APS will make every effort to support the CAT in the installation activities. These efforts will be worked out on a timely basis. 


\section{CAT Beamline Safety}

\section{Q. Will the APS establish and release specific safety mandates and guidance?}

A. Yes, the process has already begun. The Workshop of CAT Safety Representatives was held in July 1992. The APS has already issued the first of a series of CAT Safety Planning Guides. The APS is planning to provide APS-specific safety training to all the users. Special safety requirements for a specific CAT need will be considered on a one-to-one basis when the CAT indicates such needs to the APS.

\section{Q. Will there be safety training available to CAT stafi members} from the APS? How frequently will training sessions be held and what will they cover?

A. A detailed list of the safety training courses for the CAT members has not yet been finalized by the APS. The training will be based on user needs, which will be developed from user profiles.

Q. What site-specific beamline construction safety training is required for the CATs?

A. Again, stich training is now being evaluated, and the CATs will be informed of such requirements before their construction activities begin in the APS Experiment Hall. 
○

-

- 


\section{CAT Beamline Operations}

\section{Q. Where will the liquid nitrogen be available at the APS?}

A. The liquid nitrogen storage tanks will be installed outside each of the LOMs.

Q. Does the APS plan to organize a stock room?

A. Yes, the APS has planned to have a stock room in the Central Lab/Office (CLO) building. An early version of this stock room is now being organized for use by the APS staff on site.

Q. What is the location of the user shops? Will they be supervised?

A. Staff shops are planned in each of the LOMs for CAT members and other users. These shops will be supervised by the CATs.

Q. When is a CAT expected to begin providing beam time to Independent Investigators?

A. A policy on this issue has to provide enough time for esch CAT io fully commission the beamline and gain operational experience before the Independent Investigators begin their work on a beamline.

Q. At what stage during the operation will the $\mathrm{ID}$ vacuum chamber be changed from 12-mm aperture to 8-mm aperture?

A. At this time, the APS cannot predict an exact scenario for ID vacuum chamber change over. Every effort will be made to operate the storage ring with 8-mm aperture ID chambers as early as is realistic to do so, perhaps a year or so after early operations begin. Undulator users should plan their early experimental program such that it can be supported by radiation energy ranges with $12-\mathrm{mm}$ aperture chambers. 

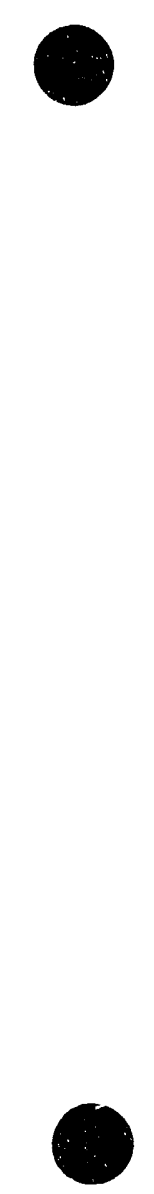


\section{Miscellaneous}

\section{Q. When can the CATs begin their work in the APS Experiment Hall?}

A. According to the current DOE funding profile for APS construction, the Experiment Hall beneficial occupancy will be in February, 1994. (CATs will be kept updated on this information.) CATs that have succe fully completed their Final Designs and safety training will be allowed to begin their construction work in the APS Experiment Hall.

\section{Q. When will the sector(s) be assigned to the CATs?}

A. After the successful completion of the Conceptual Design a che signing of an MOU, sector assignment will take place. The APS requests that CATs submit a ixy information that may be relevant to the sector assignment.

Q. When will the Iab/Office Modules (LOMs) be available to CATs?

A. According to the current DOE funding profile for the APS, four LOMs (to support 16 sectors) will be completed for beneficial occupancy in mid-1995. Soon after that date, the CATs can occupy their $1.0 \mathrm{M}$ if all interior work is complete.

Q. Who will design the interior of the LOM?

A. The laboratories inside the LOM3 will be furnished by the APS. The office space will have carpeted floors, dropped ceilings, and lights. The CAT will provide partitions in the office space, if needed, and furniture. If the partitions are permanent, the APS will carry out the construction work.

Q. When will the ANL and APS "Gate Passes" be issued to the CAT nembers?

A. As the CAT members begin intensive activity at the ANL during 1993-94, gate passes will be issued to enter ANL. Access to the APS site will be arranged as required during this period. Once the installation work begins, CAT members will have access to the APS campus. 
Q. When will CATs have information on operational costs, for example, charges for Lab/Office Module (LOM) space, telephone use, etc.

A. Because these buildings have not yet been built, it is difficult to provide eract information at this time. The LOM space assigned to a CAT occrasy ying a single sector is roughly $2400 \mathrm{sq}$. $\mathrm{ft}$. (comprising two labs and off e space). An estimate for space costs at Argonne National Laboratory is approximately $\$ 8-12 /$ sq. $\mathrm{ft}$./year.

Q. How will full-cost-recovery charges for proprietary research be calculated?

A. An exact form ila for such a calculation is not available now. The APS has provided adequate guidance on this issue to those CATs in need of such information.

Q. Will CATs need to use APS riggers to move equipment and materials on the APS site?

A. Yes, CATs will need to arrange for these services through the User Office (telephone: 708/252-9090).

Q Is space available for CATs to store equipment?

A. The APS will make every effort to provide CATs with storage space for equipment. Because such space is not yet available, we suggest that the CATs contact the APS when their need for storage space have been determined. 


\section{APPENDIX 1}

\section{Memorandum of Understanding}

Between the Advanced Photon Source and

for the Construction and Operation of Beamlines at the Advanced Photon Source

\section{Purpose}

The purpose of this Memorandum of Understanding (MOU) between the Advanced Photon Source (APS) and the Collaborative Access Team (_CAT) is to provide _CAT with access to ___ (identify specific sectors or indicate number of sectors allotted) for the construction and operation of beamlines.

\section{Prerequisites}

CAT has met the four prerequisites for signing of this MOU:

- APS approval of the _CAT proposal in accordance with the recommendations of the Proposal Evaluation Board (PEB).

- APS approval of the Conceptual Design(s) of the _CAT beamline(s).

- APS approval of the _ CAT Management Plan, the elements of which are subject to revision by mutual agreement of the parties as the project matures.

- Receipt by the APS of appropriate documentation with respect to the funding intent of the organizations identified by CAT as sources of financial support.

\section{Understanding Between the Advanced Photon Source and} CAT

During its tenure at the APS, CAT will develop and operate (identify specific sectors or indicate number of sectors allotted), and it will occupy the corresponding (or identify, if necessary) Laboratory/Office Module(s). CAT's tenure begins on the date this MOU is signed, and it may be renewed at three-year intervals after CAT receives Approval to Operate, in accordance with the provisions of the Advanced Photon Source User Policies and Procedures.

The APS and _CAT will conduct their operations in accordance with the following:

- Advanced Photon Source User Policies and Procedures.

- The approved CAT Management Plan.

- The approved Conceptual Design of the _CAT beamlines, including the insertion device(s) specified as part of that design.

- The executed Collaborative Access Team User Agreement for the Advanced Photon Source.

\section{Signatures}

Date:

CAT Director

Date:

Associate Laboratory Director for the APS 


\section{APPENDIX 2}

\subsection{Management Plan}

A "generic" management plan (MP) is provided in Appendix B. This plan may be adopted "as is," modified as needed, or replaced with a different plan. Any plan used, however, must include the following elements:

a. ACAT management organization chart, identifying the CAT director and clearly indicating where the decision-making authority resides.

b. A detailed work breakdown structure.

c. A quality assurance (QA) and quality control (QC) plan.

d. Plans for procurement and fabrication of major beam line components.

e. Funding plans for the CAT.

f. A plan for cost, schedule, and performance control.

g. A safety plan specific to the CAT.

h. Methods for facilitat ing communication among CAT members and plans for reporting activities $6 \mathrm{CAT}$ members and the APS.

In addition to the above elements, proposals for third-party national facilities must include a description of the community to be served, a validation of the commitment of that community, and a summary of "outreach" activities, such as workshops, conducted to assess and stimulate interest in such a facility.

At a later time, the management plan can be expanded to include both a beamline operation plan and an independent-investigators plan, which will identify mechanisms through which independent investigators will interact with the CAT. More detailed instructions for the preparation of a management plan are available upon request from the APS User Support and Administration Office.

From Guidelines and Forms for Proposals to Establish Collaborative Access Teams at the Advanced Photon Source, November 1990. 


\section{APPENDIX 3 \\ Draft Guidelines for the Preparation of the Preliminary Beamline Design}

The Preliminary Design of the beamline represents approximately a 30\% design level of each of the beamline components. The design permits the CAT to develop cost estimates for the construction of the beamline, as well as a realistic time line to complete the construction tasks. In developing the Preliminary Design Report, we strongly urge the CAT to exparıd on the Conceptual Design as follows:

\section{Beamline Layout}

The layout of the beamline should include all its components and should be shown on the APS defined sector. AutoCAD drawings of the sectors have been previously provided to the CATs to accomplish this job. The general layout should also include the assignment of space for electronics racks, working tables, etc. In arriving at the layout, various safety considerations should be addressed. Please refer to APS Technical Bulletin ANL/APS/TB- 9 (February 1993) for this purpose. This bulletin also presents many details on the utilities and their layout. The layout should address various aspects of safe operation of the beamline.

\section{Drawings of Components}

Drawings of the components should be adequate to assure that sufficient space has been allocated for the functioning of the beamline components. Any relevant subcomponent assembly drawings, equipment layout, partitions, elevations, cross sections, utility placement, etc., should be shown. We encourage the designers to use any of the applicable Standard Component drawings currently available from the APS Design Exchange.

\section{Work Breakdown Structure and Component Specifications}

A detailed Work Breakdown Structure (WBS) should be developed to account for each of the components that make up the beamline. Attachment 1 presents a typical WBS for a generic insertion device beamline. Specifications adequate for procurement and fabrication of each one of the WBS items must be included in this section. The specifications will include any QA and QC topics, vendor capabilities, etc.

\section{Preliminary Cost Estimates and Schedules}

For each of the WBS items, a cost estimate for its construction should be developed. Attachment 2 is a typical cost sheet that could be used for detailing the costs. Also, detailed schedules for each of the construction activities should be prepared for every WBS item based on the information at hand, and this 
could be included in the cost sheet. Various construction activities are described below.

a. Effort costs for engineering design. These activities are usually done by the scientific and engineering staff, design staff, and drafting staff.

b. Cost of procurement and/or fabrication of subcomponents. Also provide the basis of the estimate (e.g., vendor quote, catalog price, estimated from past experience, or undocumented estimate) and the date of the estimate. In developing schedules for procurement of components requiring a long lead time, an advanced plan for procurement should be developed. It is equally important for the CATs to address QA and QC needs for each of the items being procured or fabricated. We suggest that the CA'Ts assure that the vendors have a valid QA and QC capability before a contract is signed.

If an advanced plan for procurement is to be developed, the procurement department should be involved in this activity to assure that the schedules are realistic. This also permits the CAT to discuss issues related to vendor contracts, such as sole source or fixed price, with their procurement personnel.

c. Effort costs for assembly, inspection and testing of the beamline subcomponents, for installation (integration) of the entire beamline on the APS Experiment Hall floor, and for the checkout of the beamline. The work on inspection, testing, and checkout is typically done by scientists and/or engineers, while the assembly and installation is usually done by support staff, technicians, students, etc.

d. Cost of utilities (power cables, water pipes, valves, gas manifolds, communication cables, connectors, utility and cable trays, tray supports, etc.), including the costs of installation.

e. Contingency allowance. This is typically $25-30 \%$ of estimated costs of va:iou: items. If this cost cannot be separately itemized, it should be included in the cost of individual subcomponents.

\section{f. Total construction cost (sum of a through e).}

The schedule of work should include, for each relevant element of the WBS, the proposed or actual starting date and a completion date for each of the following activities: final engineering design, procurements, fabrication tasks, assembly of subcomponents and their testing, installation (or integration) of the beamline, and checkout of the beamline. The last two activities are carried out in the APS Experiment Hall. We suggest that in managing all these construction activities, the CATs could use a project management software such as MacProject Pro. This permits establishment of milestones for the activities and measurement of their progress. More sophisticated users can also use such a software for resource management and leveling. 


\section{Additional Operational Requirements}

It is important to plan the cost and schedule for furbishing the offices assigned to the CAT in the Laboratory/Office Modules (LOMs). This includes office furniture, office partitions, and special equipment in the CAT laboratories.

In addition, any special needs required for the operation of the beamline equipment should be indicated. A separate Technical Bulletin ANL/APS/TB-9 (February 1993) has been provided to the CATs and describes the standard utilities provided by the APS in each of the sectors. Examples of special needs include: local air-conditioning capability, local ventilation, exhaust for specialty or toxic gases, radioactive sample or chemical handling capability, electrical loads greater than the APS standard supplies, special telecommunication requirements, etc.

\section{Preliminary Safety Analysis}

A preliminary safety analysis should be provided for the operation of the beamline. This should include shielding requirements, safety designs, safety interlock logic, and all special needs of the beamline operations such as the capabilities to handle hazardous gases, radioactivity, bio-hazard samples, etc.

\section{R\&D Plans}

Many CATs are planning to carryout instrumentation R\&D that will lead to the design and construction of certain components in the CAT beamline. The beamline Preliminary Design should therefore include an R\&D plan and a list of R\&D milestones and schedules. The R\&D milestones for a component should be met well before the activities on its construction begin. CATs should assure that there is a consistency between R\&D schedules and construction schedules. 


\section{Attachment 1}

$\mathbf{6 . 0}$

\section{1}

5.1.1

5.1.1.1

5.1.1.2

5.1.1.3

5.1.1.4

5.1.1.5

5.1.1.6

5.1.1.7

5.1.1.8

5.1.1.9

5.1.1.10

5.1.1.11

5.1.1.12

5.1.2

5.1.2.1

5.1.2.2

5.1.2.3

5.1.2.4

5.1.2.5

5.1.2.6

5.1.2.7

5.1.2.8

5.1.2.9

5.1.2.10

5.1.2.11

5.1.2.12

5.1.2.13

5.1.2.14

5.1.2.15

5.1.3

5.1.3.1

5.1.3.2

5.1.3.3

5.1.3.4

5.1.3.5

5.1.3.6

5.1.3.7

5.1.3.8

5.1.3.9

5.1.3.10

\section{Generic CAT Work Breakdown Structure}

\section{Generic ID Beamline}

First Optics Enclosure

User Filters

Collimator

Support Structure

ID White Beam H \& V Slits

Support Structure

ID Double Crystal Monochromator

Integral Shutter and Stops

Support Structure

Dual W/M Window

Vacuum Components (Bellows, Valves, Pumps, Gauges, etc.)

Penetration Tube (Bremsstrahlung Shielded)

First Optics Enclosure Hutch

X-Ray Scattering Station

6" I.D. Pipe \& Stand

Dual W/M Window

Integral Shutter \& Stops

Support Structure

Vacuum Components (Bellows, Valves, Pumps, Gauges, etc.)

Penetration Tube (Bremsstrahlung Shielded)

$\mathrm{X}$-Ray Scattering Hutch

Shielded Cabinet (2)

Support Structure

Spectrometer

Slits

Detectors

NIM Electronics

Additional Equipment

Data Acquisition/Motion Controls

End Station

Mono Beam H \& V Slits

Support Structure

Shielded Cabinet

4" Shielded Transport (2)

4" I.D. Shielded Bellows (1)

Mono PBPM

Support Structures

Window 4" I.D.

Shielded Cabinet

Penetration Tube (Bremsstrahlung Shielded) 
5.1.3.11

5.1.3.12

5.1.3.13

5.1.3.14

5.1.3.15

5.1 .4

5.1 .5

5.1 .6

5.1 .7

5.1 .8
ID Diagnostic Station Hutch

Data Acquisition / Motion Controls

Support Structure

Diffractometer

Detectors and NIM Electronics

Personnel Safety Interface

Equipment Protection Interface

Beamline Controls

Survey and Alignment

Power and Utilities 


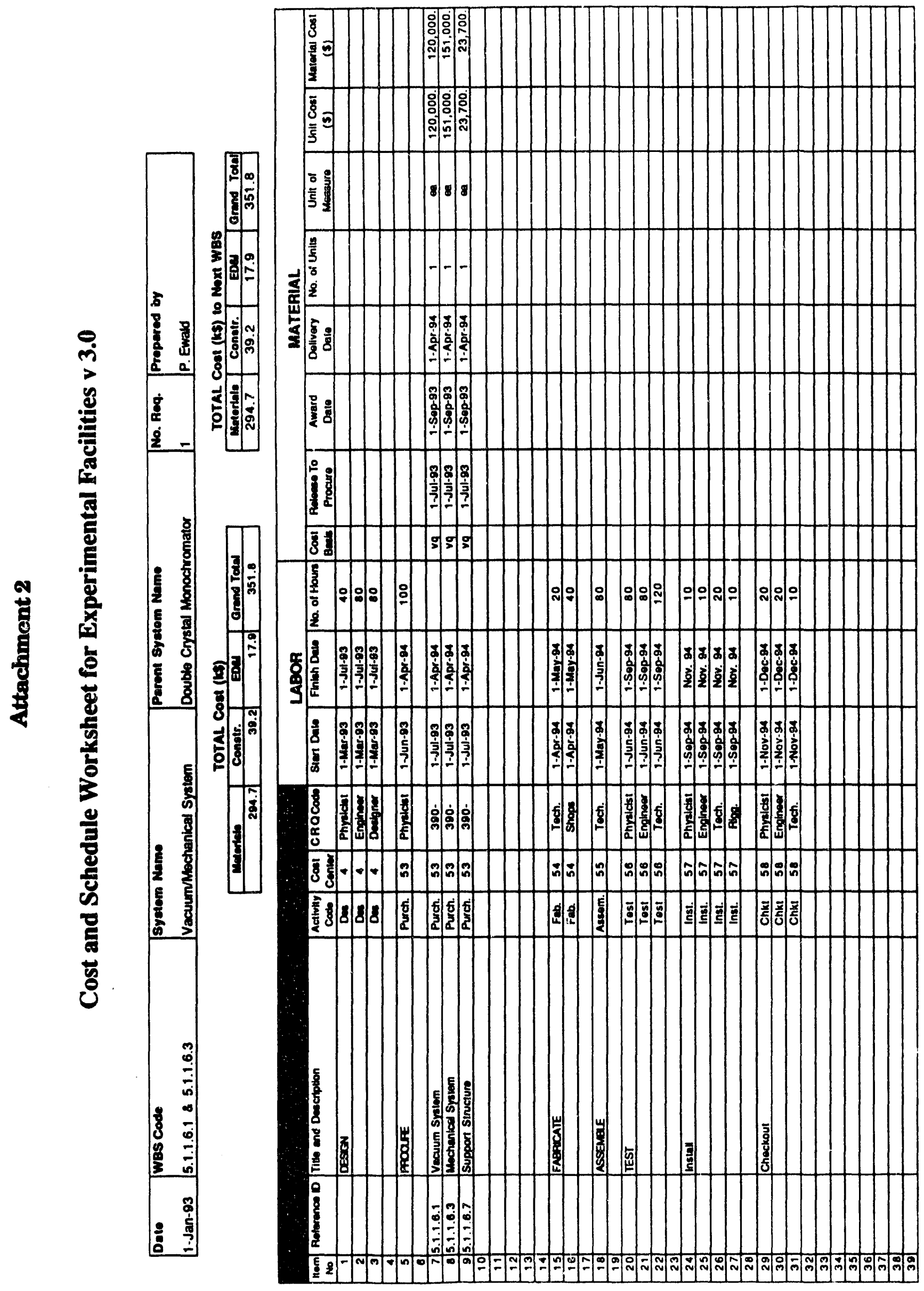




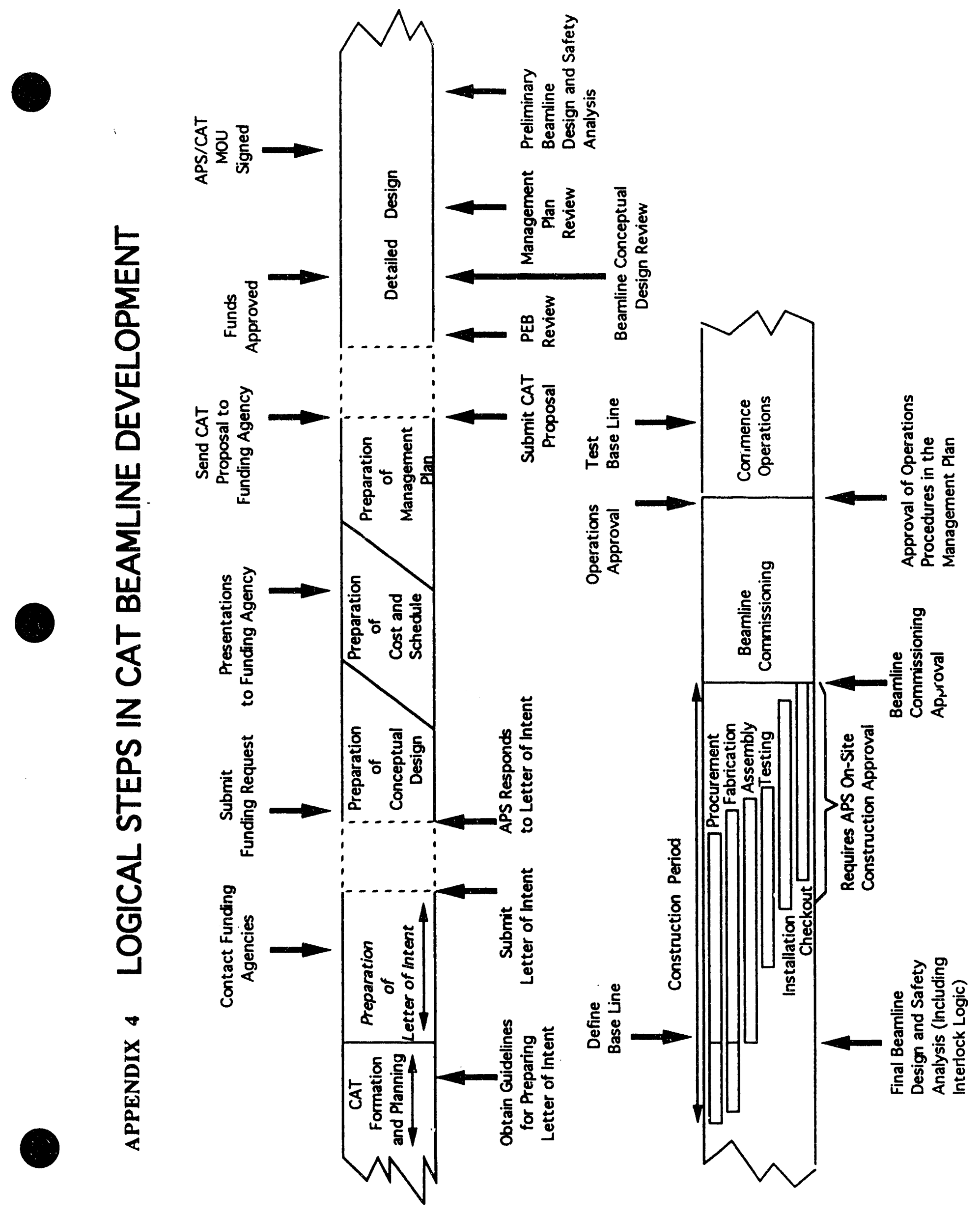



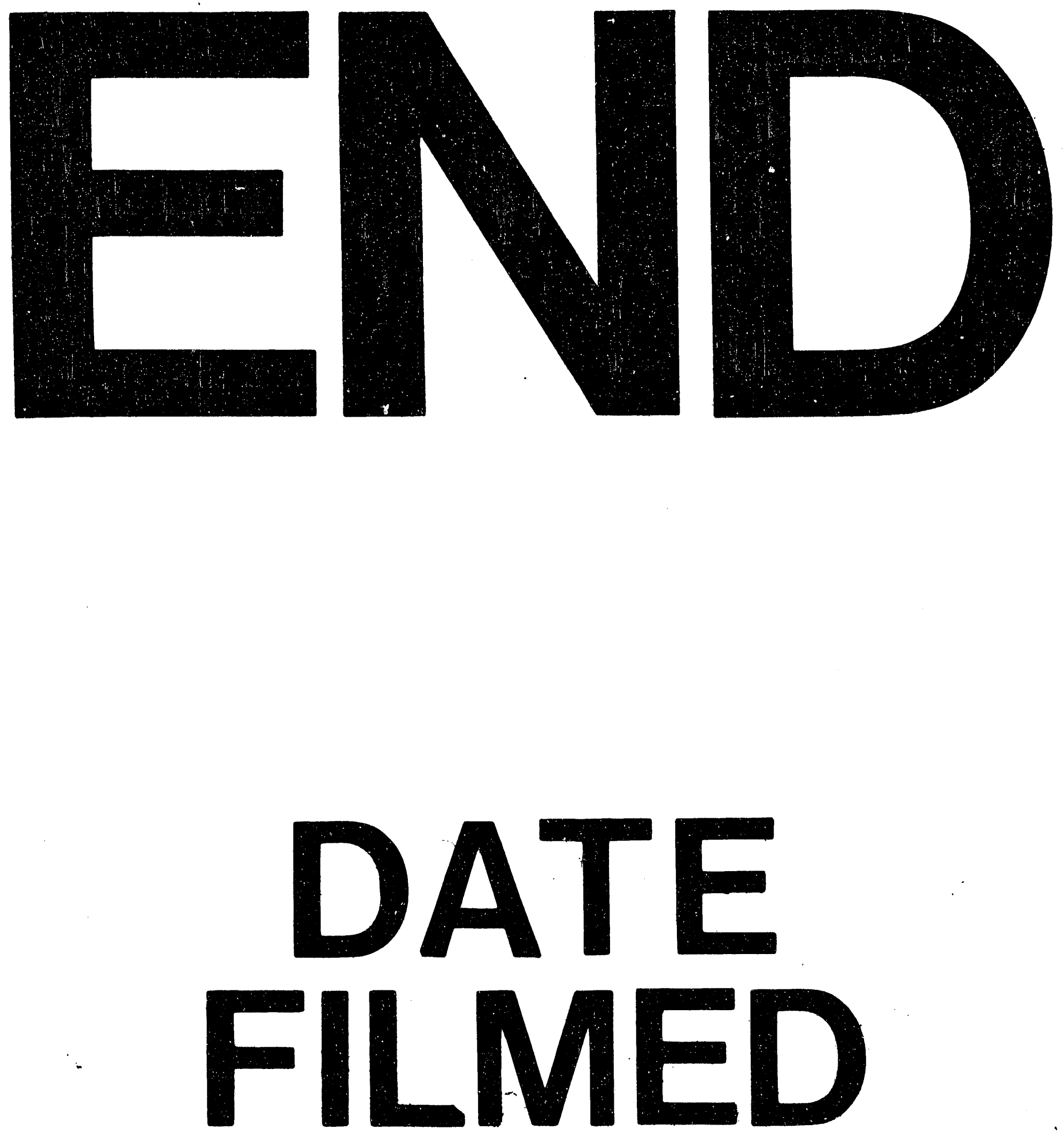

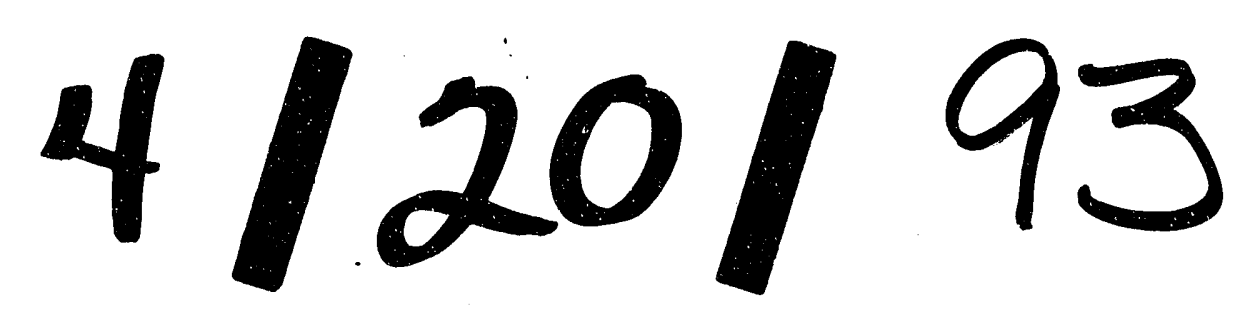


